Brief Report

\title{
Plasma Flows in Solar Filaments as Electromagnetically Driven Vortical Flows
}

\author{
Yuri E. Litvinenko (D)
}

check for updates

Citation: Litvinenko, Y.E. Plasma

Flows in Solar Filaments as Electromagnetically Driven Vortical Flows. Physics 2021, 3, 1046-1050. https://doi.org/10.3390/ physics3040065

Received: 22 August 2021 Accepted: 3 November 2021 Published: 10 November 2021

Publisher's Note: MDPI stays neutral with regard to jurisdictional clai$\mathrm{ms}$ in published maps and institutional affiliations.

Copyright: (C) 2021 by the author. Licensee MDPI, Basel, Switzerland. This article is an open access article distributed under the terms and conditions of the Creative Commons Attribution (CC BY) license (https:// creativecommons.org/licenses/by/ $4.0 /)$.
Department of Mathematics, University of Waikato, Hamilton 3105, New Zealand; yuril@waikato.ac.nz

\begin{abstract}
Electromagnetic expulsion acts on a body suspended in a conducting fluid or plasma, which is subject to the influence of electric and magnetic fields. Physically, the effect is a magnetohydrodynamic analogue of the buoyancy (Archimedean) force, which is caused by the nonequal electric conductivities inside and outside the body. It is suggested that electromagnetic expulsion can drive the observed plasma counter-streaming flows in solar filaments. Exact analytical solutions and scaling arguments for a characteristic plasma flow speed are reviewed, and their applicability in the limit of large magnetic Reynolds numbers, relevant in the solar corona, is discussed.
\end{abstract}

Keywords: space physics; plasma physics

\section{Introduction}

Solar filaments are sheets of dense and cool plasma, surrounded by the much hotter plasma of the solar corona. The relatively low temperatures and high densities of the filament material suggest that filaments are supported against gravity by a strong magnetic field $\mathbf{B}$ in the solar atmosphere [1].

Early models of solar filaments postulated static or quasi-steady equilibria and analyzed simplified magnetohydrodynamic (MHD) equations [2,3]. More recent theoretical models employed a force-free approximation, $(\nabla \times \mathbf{B}) \times \mathbf{B} \approx 0$, to successfully describe the overall filament structure [4,5].

Realistic theoretical models should satisfy a number of observational conditions for the formation and maintenance of filaments [6]. The modeling of plasma flows in filaments appears to be of particular interest in relation to their structure and evolution. Observations clearly demonstrated that even quiescent filaments are not static formations but rather are systems of jets streaming along the filaments with speeds up to $30 \mathrm{~km} \mathrm{~s}^{-1}$ [7]. Thermal nonequilibrium is typically invoked to explain the observed flows [8,9]. However, one of the puzzles of the small-scale dynamics in filaments is the physical mechanism of counterstreaming-the observed simultaneous flows with speeds of $5-20 \mathrm{~km} \mathrm{~s}^{-1}$ in opposite directions in filament barbs (feet) $[10,11]$.

The purpose of this paper is to advocate the electromagnetic expulsion force, whose effects are well-known in engineering and industrial applications, as a mechanism of counter-streaming in solar filaments.

\section{Electromagnetically Generated Vortical Flows}

Following the original argument of [12], consider an incompressible conducting fluid (plasma) with density $\rho_{0}$, temperature $T_{0}$, and electric conductivity $\sigma_{0}$ in a magnetic field $\mathbf{B}_{0}$. Provided $B_{0} \gg 4 \pi a j_{0} / c$, where $c$ is the speed of light, both $\mathbf{B}_{0}$ and the electric current density $\mathbf{j}_{0}$ can be assumed to be locally uniform ( $a$ is a typical length scale of the problem). The resulting Lorentz force is also uniform:

$$
\mathbf{f}_{0}=\frac{1}{c} \mathbf{j}_{0} \times \mathbf{B}_{0}
$$


Depending on the relative orientations of the gravity force $\rho_{0} \mathbf{g}$, where $\mathbf{g}$ is the body accelerations, and $\mathbf{f}_{0}$, the Lorentz force makes the fluid effectively heavier or lighter. In either case, the uniform volume force is potential, $\nabla \times \mathbf{f}_{0}=0$, and, just like the gravity force $\rho_{0} \mathbf{g}$, will be balanced by the pressure gradient:

$$
\nabla p_{0}=\rho_{0} \mathbf{g}+\mathbf{f}_{0}
$$

Now consider a body (filament) of volume $V$, with a temperature $T_{1} \neq T_{0}$ and the corresponding electric conductivity $\sigma_{1} \neq \sigma_{0}$, submerged in the plasma. The total force acting on the body is as follows:

$$
\mathbf{F}=\int_{V}\left(\rho_{1} \mathbf{g}+\mathbf{f}_{1}\right) \mathrm{d} V+\oint_{S} p_{0} \mathbf{n} \mathrm{d} S .
$$

Here,

$$
\mathbf{f}_{1}=\frac{1}{c} \mathbf{j}_{1} \times \mathbf{B}_{0}
$$

is the Lorentz force inside the filament, $\rho_{1}$ is the density of the filament, $\mathbf{j}_{1}$ is the current density inside the filament, $\mathbf{n}$ is the inward normal to the surface $S, p_{0}$ is the gas pressure. If the current $\mathbf{j}_{0}$ remains uniform in the presence of the body and $\mathbf{B}_{0}$ remains approximately uniform, then $\mathbf{F}$ could be expressed as

$$
\mathbf{F}=\int_{V}\left(\rho_{1} \mathbf{g}+\mathbf{f}_{1}\right) \mathrm{d} V-\int_{V} \nabla p_{0} \mathrm{~d} V,
$$

and so

$$
\mathbf{F}=\int_{V}\left(\rho_{1}-\rho_{0}\right) \mathbf{g} \mathrm{d} V+\frac{1}{c} \int_{V}\left(\mathbf{j}_{1}-\mathbf{j}_{0}\right) \times \mathbf{B}_{0} \mathrm{~d} V .
$$

Here, the first integral is the usual buoyancy (Archimedean) force. The second integral describes the electromagnetic expulsion force. It vanishes only if the electric current density remains uniform, $\mathbf{j}_{1}=\mathbf{j}_{0}$, which happens only if $\sigma_{1}=\sigma_{0}$.

Although the MHD expulsion force is formally similar to the Archimedean force in hydrodynamics, it is different from the well-known magnetic buoyancy force [13]. The magnetic buoyancy force is the usual buoyancy force, associated with the density difference caused by the pressure difference in a magnetostatic equilibrium. By contrast, the expulsion force is independent of the presence of gravity.

The key point for the following is that the static description is purely illustrative. In reality, the expulsion force will almost always drive plasma flows. As the current density is not uniform in the presence of a filament with conductivity $\sigma_{1} \neq \sigma_{0}$, the resulting Lorentz force $\mathbf{j} \times \mathbf{B} / c$ is generally not potential. Hence, it cannot be balanced by potential forces such as the gas pressure gradient $\nabla p_{0}$. This is why in general the convective term $\rho_{0}(\mathbf{v} \cdot \nabla) \mathbf{v}$, where $\mathbf{v}$ is the plasma velocity, and the viscous term $\eta \nabla^{2} \mathbf{v}$, where $\eta$ is the scalar viscosity, must be taken into account in the equation of motion. Physically, this means that electromagnetically generated vortical flows must appear in the vicinity of a submerged body [12]. It is these flows that may naturally explain the plasma counter-streaming in solar filaments. Assuming an incompressible steady plasma flow, the equation of motion is as follows:

$$
\rho_{0}(\mathbf{v} \cdot \nabla) \mathbf{v}=-\nabla p+\rho_{0} \mathbf{g}+\eta \nabla^{2} \mathbf{v}+\frac{1}{c} \mathbf{j} \times \mathbf{B} .
$$

The electromagnetic expulsion force, also known as electro-magneto-phoresis, has been extensively studied under laboratory conditions, motivated by such engineering applications as the extraction of impurities in liquid metals and the separation of mechanical mixtures and biological cells [14-16]. 


\section{Counter-Streaming in Solar Filaments}

Although the parameter regime in the solar atmosphere differs significantly from that under laboratory conditions, the expulsion force might play a role in the filament dynamics. Solar filaments consist of numerous fine and dense threads with a typical radius $a \approx 10^{7} \mathrm{~cm}$ and temperature $T_{1} \approx 10^{4} \mathrm{~K}[1]$. For the coronal temperature $T_{0} \approx 10^{6} \mathrm{~K}$, the ratio of conductivities inside and outside the filament is

$$
\frac{\sigma_{1}}{\sigma_{0}}=\left(\frac{T_{1}}{T_{0}}\right)^{3 / 2} \approx 10^{-3} \ll 1 .
$$

As the electric current density $j_{1} \ll j_{0}$, expected effects of the expulsion force in filaments should be significant.

The calculation of the general expressions for the electromagnetic expulsion force and the associated vortical flows is a complicated nonlinear problem. Several particular cases, however, can be studied in detail. Analytical progress was achieved in the limit of small ordinary $\operatorname{Re}=\rho_{0} v_{0} a / \eta$ and magnetic $\operatorname{Re}_{m}=4 \pi \sigma_{0} v_{0} a / c^{2}$ Reynolds numbers and a small Hartmann number $M=\left(a B_{0} / c\right)\left(4 \pi \sigma_{0} / \eta\right)^{1 / 2}$. In this limit, the convective derivative can be ignored in the equation of motion, the electromagnetic and dynamic problems decouple, and exact analytical expressions for the plasma flows and the expulsion force can be obtained for a sphere and a cylinder with radius $a[12,17]$. Further analytical progress was achieved for particles of other shapes by using symmetry considerations and asymptotic methods [18,19].

As a potentially relevant example, consider a solution to the problem of electromagnetically driven flows near a cylinder with radius $a$ and electric conductivity $\sigma_{1}$ surrounded by the plasma with conductivity $\sigma_{0} \gg \sigma_{1}$ and viscosity $\eta$, given the uniform electric current $\mathbf{j}_{0}=\left(j_{\perp}, 0, j_{\|}\right)$and an approximately uniform magnetic field $\mathbf{B}_{0}=\left(B_{x}, B_{y}, B_{z}\right)$ outside the cylinder. If the cylinder is co-aligned with the $z$-axis, the solution for the plasma velocity outside the cylinder $(r>a)$ is as follows:

$$
\begin{gathered}
v_{x}=0, \quad v_{y}=0, \\
v_{z}(r, \phi)=\frac{a^{2} j_{\perp}}{4 c \eta}\left(B_{x} \sin 2 \phi-B_{y} \cos 2 \phi\right)\left(1-\frac{a^{2}}{r^{2}}\right) .
\end{gathered}
$$

Here, $r=\left(x^{2}+y^{2}\right)^{1 / 2}$ and $\phi=\tan ^{-1}(y / x)$ is the polar angle (the counterclockwise angle from the $x$-axis in the $x y$-plane). The solution for a viscous fluid satisfies the standard boundary condition of vanishing velocity on the surface of the cylinder [17]. The expression for $v_{z}(r, \phi)$ is basically a product of two functions: $\left(B_{x} \sin 2 \phi-B_{y} \cos 2 \phi\right)$ describes the alternating flow directions in the neighboring sectors of the $x y$-plane, whereas $\left(1-a^{2} / r^{2}\right)$ for $r>a$ describes a monotonic increase in the speed with distance from the surface of the cylinder.

Two points are worth stressing. First, the trigonometric dependence of $v_{z}$ on the polar angle $\phi$ means that the oppositely directed flows are naturally predicted by the model. Second, the flows are generated even when the external magnetic field and electric current are co-aligned $\left(B_{y}=0\right.$ and $\left.j_{\perp} B_{z}=j_{\|} B_{x}\right)$, corresponding to a large-scale force-free magnetic field in the solar corona, $\mathbf{j} \times \mathbf{B}=0$. This is a clear illustration of how the action of the expulsion force differs from that of the standard $\mathbf{j} \times \mathbf{B}$ force.

The presented velocity profile provides strong motivation for modeling counterstreaming in solar filaments as electromagnetically generated plasma flows. As far as characteristic values of the key dimensionless parameters are concerned, for typically observed flow speeds in solar filaments of order $v_{0} \approx 10 \mathrm{~km} \mathrm{~s}^{-1}$, the ordinary Reynolds number is of the order $10^{-2}$, and thus $\operatorname{Re} \ll 1$ as in the solution above. By contrast, the magnetic Reynolds number is large, $\operatorname{Re}_{m} \simeq 10^{9} \gg 1$. This is why the solution above cannot be directly applied to describe flows along the filament threads in the solar corona. Dimensional arguments, however, suggest a simple formula for the expulsion force density: 


$$
\mathbf{f}=-\mathbf{f}_{0} \Phi\left(\operatorname{Re}, \operatorname{Re}_{m}\right) .
$$

The dimensionless function $\Phi$ has the following asymptotic behavior [20]:

$$
\Phi \approx \begin{cases}1, & \operatorname{Re}_{m}<1 \\ 1 / \operatorname{Re}_{m}, & \operatorname{Re}_{m}>1 .\end{cases}
$$

These expressions lead to an estimate for the typical plasma speed $v_{0}$, which is consistent with observations of counter-streaming in solar filaments in the limit $\operatorname{Re}_{m} \gg 1$ [21].

To put the qualitative dimensional arguments on a firmer footing, it would be necessary to derive an expression for the plasma velocity in the vicinity of a cylindrical filament thread, which would remain valid for both small and large values of $\operatorname{Re}_{m}$. Symmetry dictates that the sought-after solution in cylindrical geometry is independent of $z$ if the cylinder is oriented along the $z$-axis, in which case MHD equations reduce to two secondorder partial differential equations with the only parameter being the Hartmann number $M$. Solutions of the resulting boundary value problem for particular orientations of the magnetic field have been constructed [22]. Further analytical progress for arbitrary orientations of the electric current and magnetic field near the filament may be achieved by deriving asymptotic analytical solutions in the limits of small and large $M$ and by matching the solutions for $M \approx 1$.

\section{Discussion}

Theoretical and numerical studies of solar filaments traditionally consider idealized, nearly force-free magnetic field configurations $[4,5]$. The boundary conditions are controlled by the photospheric plasma flows and magnetic flux emergence. The gas pressure gradient and the gravity force are among the local forces acting on a filament in the corona, whose effects can be described by perturbing a large-scale force-free model [23].

Whereas the equilibrium in filaments is primarily determined by a balance between the Lorentz and gravity forces, the observed counter-streaming remains an unsolved problem [24]. It is worth stressing that even quiescent filaments are highly dynamic formations, characterized by mass flows and changes of shape on multiple scales [7].

As argued above, what appears to be missing in the available models of solar filaments is an evaluation of the role of the electromagnetic expulsion force in the filament dynamics. The expulsion force is an MHD analogue of the usual buoyancy (Archimedean) force $[12,25]$, which provides a well-known method for engineering applications such as impurity extraction and the separation of bioparticles [16,26]. A nonuniform distribution of temperature in filaments leads to a nonuniform electric conductivity and hence to a nonuniform Lorentz force. The resulting electromagnetic expulsion force is generally nonpotential and naturally drives vortical plasma flows that may correspond to the observed counter-streaming flows in filaments [21].

Since the model calculation of the counter-streaming flows is performed in an idealized geometry, it is difficult to speculate whether the model might predict different features for the flows in different parts of a filament. It is reasonable to expect though that, as long as the flows are generated in filament barbs, they should also be sustained in the main body of the filament (along the filament spine) whose mass is supported against gravity by a magnetic force.

The proposed mechanism may provide an explanation for the observed counterstreaming in filaments. More generally, the electromagnetic expulsion force may play a role in the dynamics of cosmic plasma with nonuniform distributions of temperature and electric conductivity. 
Funding: This research was partly funded by the Deutsche Forschungsgemeinschaft (project number 434200803).

Acknowledgments: It is a pleasure to contribute to this Special Issue, dedicated to Professor Reinhard Schlickeiser, who was my host during several visits to Ruhr-Universität Bochum, supported by a Humboldt Foundation fellowship between 2007 and 2011.

Conflicts of Interest: The author declares no conflict of interest.

\section{References}

1. Tandberg-Hanssen, E. The Nature of Solar Prominences; Kluwer Academic Publishers: Dordrecht, The Netherlands, 1995. [CrossRef]

2. Kippenhahn, R.; Schlüter, A. Eine Theorie der solaren Filamente. Zeit. f. Astrophys. 1957, 43, 36-62. Available online: http: / / adsabs.harvard.edu/full/1957ZA.....43...36K (accessed on 15 August 2021).

3. Brown, A. On the stability of a hydromagnetic prominence model. Astrophys. J. 1958, 128, 646-663. [CrossRef]

4. Aulanier, G.; Démoulin, P. 3-D magnetic configurations supporting prominences. I. The natural presence of lateral feet. Astron. Astrophys. 1998, 329, 1125-1137. Available online: http:/ / adsabs.harvard.edu/full/1998A\&A...329.1125A (accessed on 15 August 2021).

5. Gibson, S.E. Solar prominences: theory and models. Fleshing out the magnetic skeleton. Living Rev. Sol. Phys. 2018, 15, 7. [CrossRef]

6. Martin, S.F. Conditions for the formation and maintenance of filaments. Sol. Phys. 1998, 182, 107-137. [CrossRef]

7. Wang, Y.-M. The jetlike nature of He II $\lambda 304$ prominences. Astrophys. J. 1999, 520, L71-L74. [CrossRef]

8. Craig, I.J.D.; McClymont, A. N. Quasi-steady mass flows in coronal loops. Astrophys. J. 1986, 307, 367-380. [CrossRef]

9. Klimchuk, J.A.; Luna, M. The role of asymmetries in thermal nonequilibrium. Astrophys. J. 2019, 884, 68. [CrossRef]

10. Diercke, A.; Kuckein, C.; Verma, M.; Denker, C. Counter-streaming flows in a giant quiet-Sun filament observed in the extreme ultraviolet. Astron. Astrophys. 2018, 611, A64. [CrossRef]

11. Zirker, J.B.; Engvold, O.; Martin, S.F. Counter-streaming gas flows in solar prominences as evidence for vertical magnetic fields. Nature 1998, 396, 440-441. [CrossRef]

12. Leenov, D.; Kolin, A. Theory of electromagnetophoresis. I. Magnetohydrodynamic forces experienced by spherical and symmetrically oriented cylindrical particles. J. Chem. Phys. 1954, 22, 683-688. [CrossRef]

13. Parker, E.N. The formation of sunspots from the solar toroidal field. Astrophys. J. 1955, 121, 491-507. [CrossRef]

14. Kolin, A. Some current and potential uses of magnetic fields in electrokinetic separations. J. Chromatogr. 1978, 159, 147-181. [CrossRef]

15. Terada, T.; Akiyama, Y.; Izumi, Y.; Nishijima, S. Separation of impurity in molten metals by using superconducting magnet. Phys. C 2009, 469, 1845-1848. [CrossRef]

16. Watarai, H.; Suwa, M.; Iiguni, Y. Magnetophoresis and electrophoresis of microparticles in liquids. Anal. Bioanal. Chem. 2004, 378, 1693-1699. [CrossRef]

17. Marty, P.; Alemany, A. Écoulement dû à des champs magnétique et électrique croisés autour d'un cylindre de conductivité quelconque. J. Mec. Theor. Appl. 1983, 2, 227-243.

18. Moffatt, H.K.; Sellier, A. Migration of an insulating particle under the action of uniform ambient electric and magnetic fields. Part 1. General theory. J. Fluid Mech. 2002, 464, 279-286. [CrossRef]

19. Yariv, E.; Miloh, T. Electro-magneto-phoresis of slender bodies. J. Fluid Mech. 2007, 577, 331-340. [CrossRef]

20. Litvinenko, Y.E.; Somov, B.V. Electromagnetic expulsion force in cosmic plasma. Astron. Astrophys. 1994, 287 , L37-L40.

21. Litvinenko, Y.E.; Somov, B.V. Aspects of the global MHD equilibria and filament eruptions in the solar corona. Space Sci. Rev. 2001, 95, 67-77. [CrossRef]

22. Gerbeth, G.; Thess, A.; Marty, P. Theoretical study of the MHD flow around a cylinder in crossed electric and magnetic fields. Eur. J. Mech. B/Fluids 1990, 9, 239-257.

23. Litvinenko, Y.E.; Wheatland, M.S. A simple dynamical model for filament formation in the solar corona. Astrophys. J. 2005, 630, 587-595. [CrossRef]

24. Chen, P.-F.; Xu, A.-A.; Ding, M.-D. Some interesting topics provoked by the solar filament research in the past decade. Res. Astron. Astrophys. 2020, 20, 166. [CrossRef]

25. Ščepanskis, M.; Jakovičs, A. The magnetohydrodynamic force experienced by spherical iron particles in liquid metal. J. Magn. Magn. Mater. 2016, 403, 30-35. [CrossRef]

26. Xuan, X. Recent advances in continuous-flow particle manipulations using magnetic fluids. Micromachines 2019, 10, 744. [CrossRef] 